\title{
Medición del Bienestar Subjetivo y Condiciones de Vida en el contexto sociocultural de la contingencia por COVID en Veracruz, México
}

\author{
Measurement of Subjective Well-being and living conditions in the \\ sociocultural context of the COVID contingency at Veracruz México
}

León Felipe Beltrán Guerra ${ }^{a}$-Jorge Luis Arellanez Hernández Erika Cortés Flores ${ }^{\mathrm{d}}-$ Martha Elba Ruiz Libreros ${ }^{\mathrm{e}}$

a Observatorio de Calidad de Vida y Salud Social, Instituto de Investigaciones Psicológicas, Universidad Veracruzana, Xalapa, México.

Contacto: lebeltran@uv.mx

b Observatorio de Calidad de Vida y Salud Social, Instituto de Investigaciones Psicológicas, Universidad Veracruzana, Xalapa, México.

Contacto: jarellanez@uv.mx

c Observatorio de Calidad de Vida y Salud Social, Instituto de Investigaciones Psicológicas, Universidad Veracruzana, Xalapa, México.

Contacto: eromero@uv.mx

d Observatorio de Calidad de Vida y Salud Social, Instituto de Investigaciones Psicológicas, Universidad Veracruzana, Xalapa, México.

contacto: ecortes@uv.mx

e Observatorio de Calidad de Vida y Salud Social, Instituto de Investigaciones Psicológicas,

Universidad Veracruzana, Xalapa, México.

Contacto: maruiz@uv.mx

Recibido: 03 de septiembre de 2020

Aceptado: 15 de octubre de 2020 
RESUMEN: En la actualidad, resulta cada vez más relevante estudiar el concepto de Bienestar desde una perspectiva multidimensional, que considere no sólo variables macrosociales, sino también aquellas inherentes a los individuos, como es el bienestar subjetivo. Desde el Observatorio de Calidad de Vida y Salud Social (OCVS), se ha aplicado una encuesta en línea con el objetivo de describir los niveles de bienestar subjetivo en población adulta del Estado de Veracruz en el contexto de la contingencia actual por COVID-19, con la finalidad de identificar las áreas de atención prioritarias para su intervención a corto y mediano plazo. Se realizó un estudio no experimental, ex post facto, con un muestreo por conveniencia, en el que se utilizó la escala Bienestar Subjetivo y Condiciones de Vida (BISCOV), participaron 485 personas, mayores de 18 años, radicadas en el estado de Veracruz, México. Se identificó una disminución en los niveles de bienestar subjetivo y las áreas que afectan dichas valoraciones, en comparación con lo observado en mediciones previas. Esto permite sentar una base empírica de las repercusiones que está permeando la pandemia por el COVID-19 en la vida cotidiana de los participantes en el estudio.

Palabras clave: Bienestar subjetivo; condiciones de vida; calidad de vida.

ABSTRACT: The study Well-being from a multidimensional perspective must considers not only macro variables, but also those inherent to individuals, such as subjective well-being. From the Observatory of Quality of Life and Social Health, a measurement is proposed with the objective of describing the levels of subjective well-being in the adult population of the State of Veracruz in the context of the current contingency due to COVID-19, in order to identify the priority areas of attention for their intervention in the short and medium term. A non-experimental, ex post facto study was carried out, with a convenience sampling, the Subjective Wellbeing and Living Conditions scale (BISCOV) was used, 485 people participated, over 18 years of age, based in the state of Veracruz, Mexico. A decrease in the levels of subjective well-being and the areas that affect these evaluations was identified.

Keywords: Subjective Well-being; Living Conditions; Quality of life.

\section{Introducción}

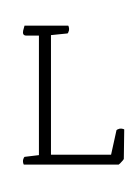

os estudios orientados a describir los niveles de bienestar de las personas cada vez toman mayor relevancia en México y en el mundo, no sólo porque es una solicitud expresa por parte de la Organización de las Naciones Unidas en la Agenda 2030 y en los Objetivos del Desarrollo Sostenible (ONU, 2018), sino porque dicha información permite a 
las instituciones del Estado y a la misma sociedad civil, generar acciones que incidan en mejorar los niveles de bienestar de la población. De manera paralela, las condiciones socioeconómicas y políticas que se desarrollan en México, así como las condiciones de pobreza, la crisis económica, las dificultades para acceso a la educación y salud, son variables que inevitablemente afectan las condiciones de bienestar y calidad de vida de las personas (Nussbaum y Sen, 2000).

Bajo esta perspectiva, los estudios a nivel mundial que abordan el Bienestar desde una perspectiva multidimensional, con variables inherentes al individuo son cada vez más frecuentes, lo que permite una mayor comprensión del fenómeno. De acuerdo con algunos autores (Sen, Stiglitz y Fitoussi, 2009; Rojas, 2011; 2012) existe una tendencia en la realización de investigaciones con esta perspectiva, consideran variables económicas y de manera paralela variables relativas a la forma en que los individuos perciben y construyen su realidad, satisfacen sus necesidades, emiten juicios a partir de ello, y emociones derivadas de tales procesos cognitivos y afectivos, integrados en el concepto de Bienestar Subjetivo.

Y así como las tendencias en los estudios del bienestar coinciden en que éstos deben elaborarse desde una perspectiva multidimensional, se coincide en que los indicadores macroeconómicos ya no son suficientes para la toma de decisiones que incida favorablemente en la vida de las personas y los contextos socioeconómicos en que se desenvuelven; de allí la propuesta de generar información sistematizada desde los destinatarios, desde la gente, con la intención de relacionar los resultados con su realidad.

Como parte del trabajo del Observatorio de Calidad de Vida y Salud Social (OCVS) ${ }^{1}$ se ha desarrollado una metodología que permite, a través de la Escala de Bienestar Subjetivo y Condiciones de Vida (BISCOV), identificar los niveles de bienestar subjetivo de la población. El

\footnotetext{
${ }^{1}$ El OCVS pertenece a la Universidad Veracruzana, y tiene como finalidad generar información centrada en el estudio de variables que incidan en el Bienestar subjetivo, la Calidad de Vida y la Salud Social de las personas que habitan en el estado de Veracruz, México y el Mundo; considerando constructos, variables e indicadores a nivel nacional e internacional que describan los diferentes atributos y dimensiones, con el objetivo de evaluar los niveles de bienestar, de manera directa o indirecta, que la gente presenta.
} 
constructo se basa en las siguientes premisas teóricas y se puede visualizar en la figura 1 (Beltrán, 2018):

- El Bienestar subjetivo está compuesto por un componente emocional y otro cognitivo, los cuales interactúan como resultado de los procesos perceptivos al momento de que un individuo se relaciona con su entorno. Ambos componentes influyen en los procesos psicológicos que permean las interpretaciones de la realidad del individuo y que regirán las valoraciones de bienestar o malestar subjetivo.

- Tales valoraciones, se verán afectadas por las diferentes dimensiones que componen el bienestar subjetivo, las cuales pueden a su vez dividirse en dos grandes grupos: aquellas relacionadas con una valoración a nivel personal (Felicidad y Satisfacción con la Vida), y en segundo plano, aquellas relacionadas con una valoración del individuo al momento de interactuar con ciertos grupos, organizaciones e instituciones de su entorno (Bienestar personal, Satisfacción con los Dominios de vida, Satisfacción con los bienes y servicios públicos).

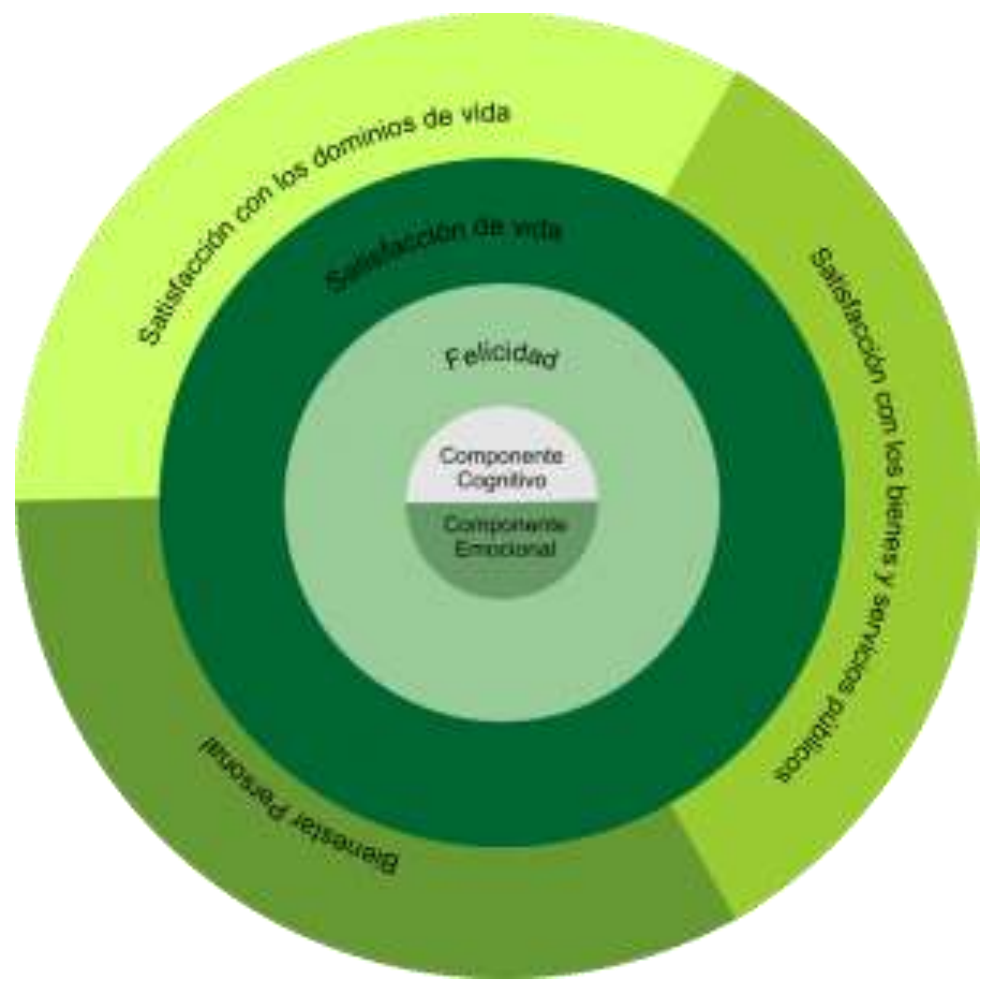

Figura 1: Componentes teóricos del Bienestar Subjetivo. Fuente: Beltrán Guerra, L.F. (2018). La psicología social en la medición del bienestar subjetivo para gestionar el desarrollo de las sociedades. Psicología Social y Cultura. México: Universidad Veracruzana 
De manera particular, el año 2020 tomando en cuenta la contingencia derivada del COVID-19, es posible identificar algunas repercusiones que pueden presentarse en las personas los niveles de bienestar percibidos, donde la crisis de salud y económica, tanto globales como locales, han generado condiciones donde los comportamientos cotidianos en el área de salud, las actividades económicas, las interacciones sociales, recreativas, deportivas, de ocio, entre muchos otros, han sido modificados.

Aunado a ello, la inmersión de la población, en mayor o menor medida, a los contextos digitales a partir del uso de tecnologías para interactuar o participar en interacciones sociales, económicas, recreativas y de salud, ha traído consigo comportamientos adaptativos con repercusiones en la salud mental, y con ello en las condiciones de bienestar de los individuos y sus entornos sociales; es decir, a su calidad de vida.

Es por ello por lo que el OCVS, se planteó como objetivo describir los niveles de bienestar subjetivo en población adulta del Estado de Veracruz en el contexto de la contingencia actual por COVID-19, con la finalidad de identificar las áreas de atención prioritarias para su intervención a corto y mediano plazo.

\section{Metodología}

Se realizó un estudio no experimental, ex post facto, con un muestreo por conveniencia, donde se aplicó una encuesta online utilizando la escala Bienestar Subjetivo y Condiciones de Vida (BISCOV). Participaron 485 personas mayores de 18 años, radicadas en el estado de Veracruz, México. El levantamiento de información se llevó a cabo durante el mes de junio de 2020 a través de medios digitales como el Website del OCVS, las redes sociales del OCVS (Facebook y Twitter), la cual se difundió a su vez en las redes sociales de los participantes. La encuesta cuidó tomar en cuenta las normas éticas de la investigación en seres humanos, tomando como elemento central el cuidado del anonimato y la confidencialidad a través del consentimiento informado (Secretaría de Salud 2013a, 2013b; Sociedad Mexicana de Psicología, 2009).

La escala BISCOV está integrada de 31 reactivos con un formato de respuesta tipo Likert considerando los factores: Felicidad y Satisfacción con la Vida (4 preguntas), Bienestar 
personal (14 preguntas), Satisfacción con los Dominios de vida (5 reactivos), y Satisfacción con los bienes y servicios públicos (8 reactivos), como lo establece el constructo de Bienestar mencionado, arrojando una valoración entre uno y cinco puntos, donde uno es el nivel más bajo de bienestar y cinco el nivel más alto.

\section{Resultados}

Como se puede apreciar en la Figura 2, los resultados relacionados con las esferas personales (Felicidad y Satisfacción con la vida) son los que reciben una valoración más alta por parte de los encuestados.

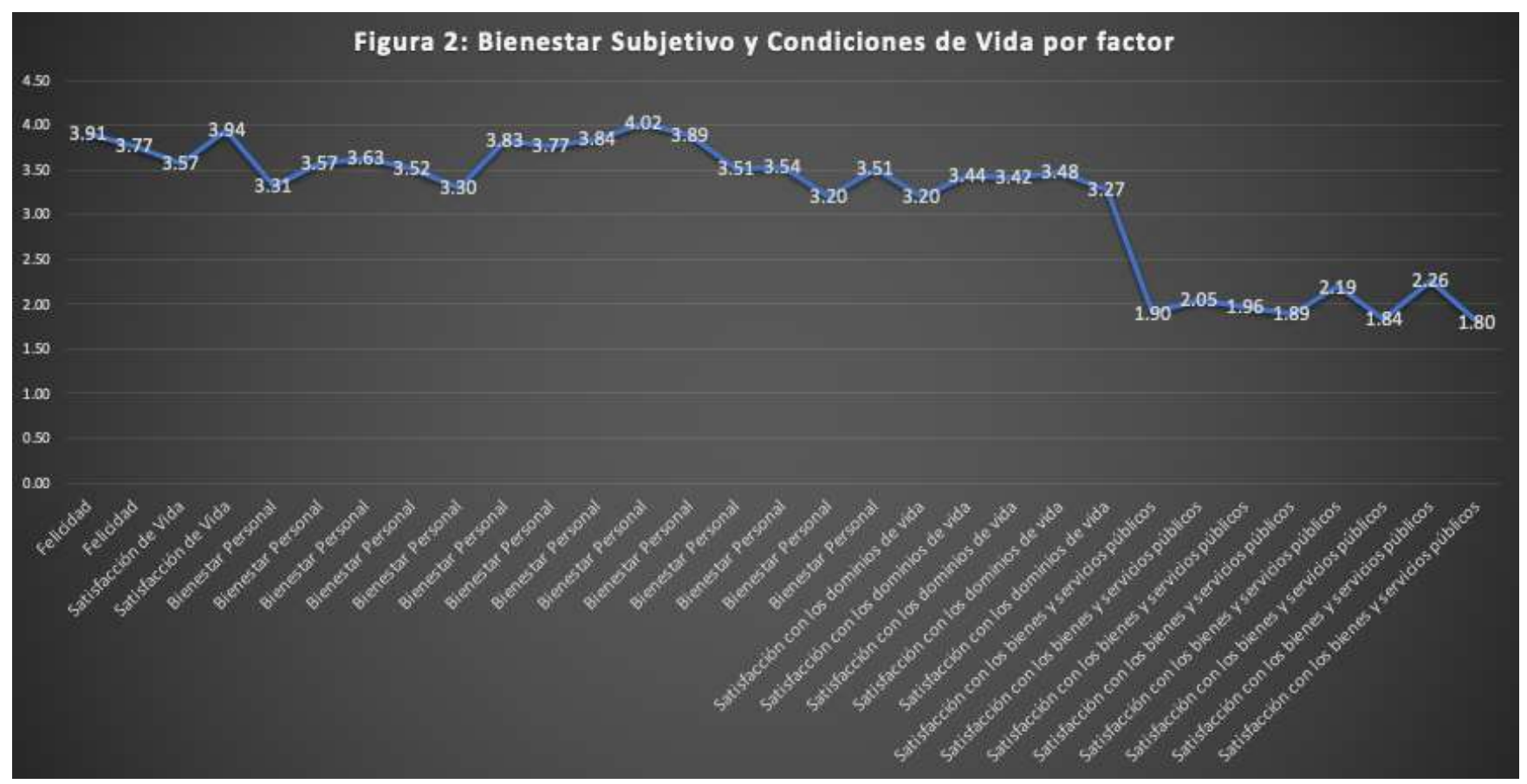

Figura 2: Bienestar Subjetivo y Condiciones de Vida por factor. Fuente: Creación propia

Conforme avanza el instrumento para explorar las demás esferas y cuestionar sobre las valoraciones que tienen que ver con las interacciones del individuo con su contexto social (Bienestar personal, Satisfacción con los dominios de vida), las calificaciones disminuyen ligeramente a un nivel MEDIO de percepción de bienestar. Se destaca que, el factor relacionado con la interacción con los bienes y servicios públicos es el que recibe la valoración más baja por parte de la población encuestada. 
Cabe destacar que los factores evaluados pertenecientes al área donde el individuo interactúa con los diversos espacios en que se desenvuelve, se relacionan con indicadores de Desarrollo Humano consideradas por el IDH (salud, educación, trabajo) del Programa de las Naciones Unidad para el Desarrollo (PNUD, 2020)

La disminución en la percepción de bienestar subjetivo puede apreciarse también en la Tabla 1, donde se identifica al factor de Felicidad como el más alto; sin embargo, la valoración general se ve afectada por las condiciones del contexto donde es posible apreciar una menor satisfacción con las áreas relativas a los dominios de vida, y mucho menor la satisfacción con los bienes y servicios públicos. Si bien, las personas se mantienen en niveles de felicidad y satisfacción más o menos estables, las condiciones de vida y el contexto en el que se encuentran limitan, de acuerdo con sus respuestas, su bienestar.

\section{Tabla 1:}

Promedio de calificaciones por factor

\begin{tabular}{|l|c|}
\hline \multicolumn{1}{|c|}{ Factor } & Calificación promedio \\
\hline Felicidad & 3.84 \\
\hline Satisfacción de Vida & 3.76 \\
\hline Bienestar Personal & 3.60 \\
\hline Satisfacción con los dominios de vida & 3.36 \\
\hline Satisfacción con los bienes y servicios públicos & 1.99 \\
\hline
\end{tabular}

Fuente: Creación propia

Con la intención de mostrar la disminución en los niveles de bienestar subjetivo y condiciones de vida, se tomó como referencia el estudio realizado en el año 2017, en el que, con una metodología y tamaño de muestra similar (n=515) y utilizando el mismo instrumento (Beltrán, Arellanez, Romero, 2017), se observan calificaciones más bajas en la percepción del bienestar. 


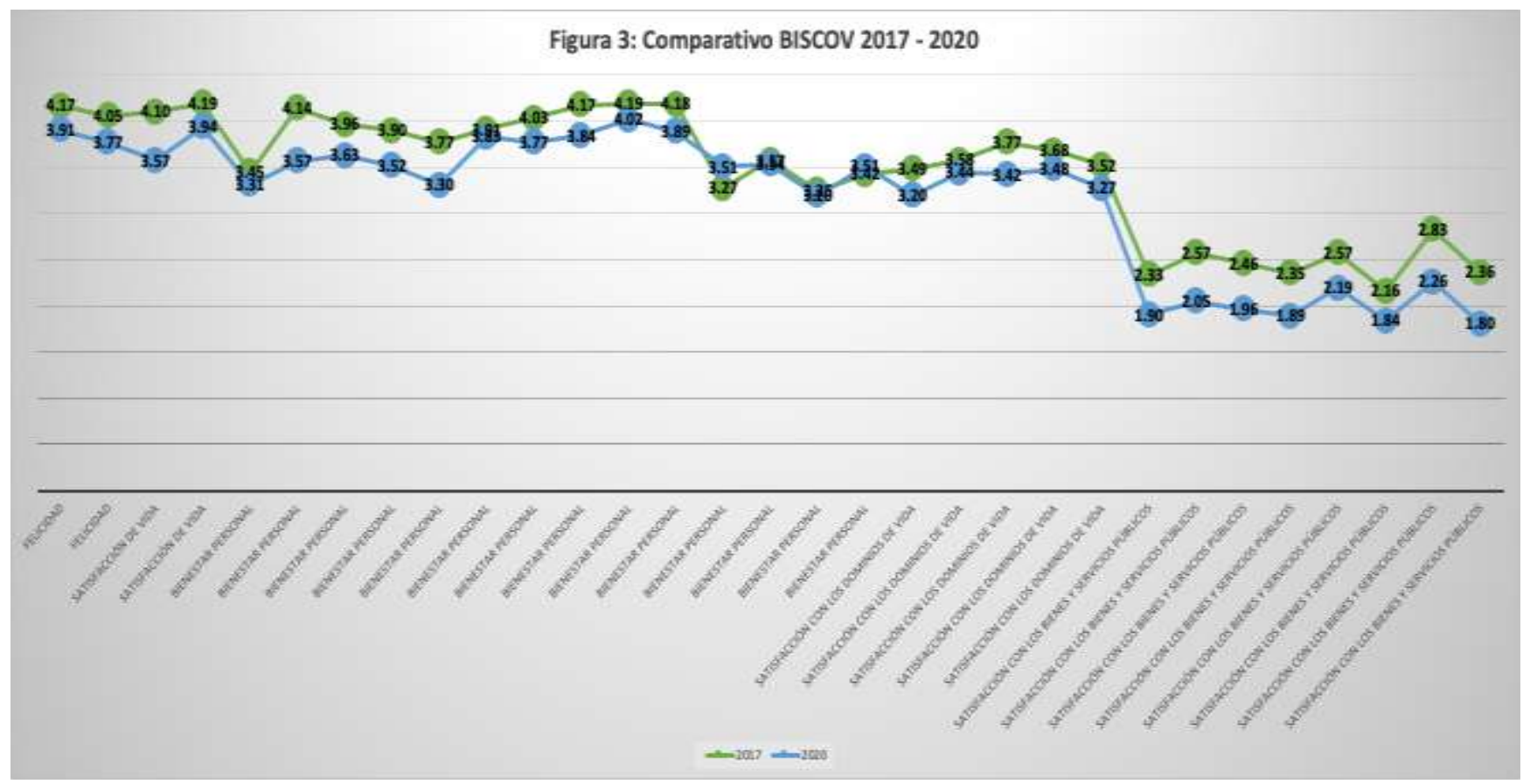

Figura 3: Comparativo BISCOV 2017-2020. Fuente: Creación propia.

Pareciera ser que la disminución en los niveles de bienestar subjetivo, de acuerdo por lo reportado por los encuestados, estarían relacionados con la situación actual del confinamiento y las repercusiones en la vida social y económica que ha acarreado el COVID19. Como se observa en la gráfica, las áreas que tienen que ver con la situación económica, donde la falta de percepción de estabilidad por el trabajo y los ingresos, así como los bienes y servicios públicos, disminuyen de manera general el nivel de Bienestar Subjetivo.

\section{Conclusiones}

Los resultados obtenidos en este estudio son consistentes con lo que otros estudios han reportado con relación a la felicidad de los mexicanos, como por ejemplo los citados por el Happy Planet Index (2020); sin embargo, esto no significa que dicha percepción sea determinante para los niveles de bienestar subjetivo, el cual, como fenómeno multidimensional, debe considerar otras dimensiones, particularmente aquellas donde las interacciones sociales y el contexto socioeconómico y cultural inciden en los niveles de desarrollo y la calidad de vida. 
La propuesta del Observatorio de Calidad de Vida y Salud Social, en ningún momento pretende ser un sustituto de los índices e indicadores de bienestar que hoy en día existen, sino más bien, que pueda sumarse para complementar la información relativa.

Sin duda, los niveles de bienestar se han visto mermados debido a las crisis económicas y de salud, áreas en las que habrá que empezar a trabajar para crear condiciones que permitan a las personas, como individuos y como sociedad, recuperar esos niveles de bienestar que les permitan retomar la estabilidad en sus vidas. El incremento en los indicadores macroeconómicos como el Producto Interno Bruto o la Taza de Ocupación no son suficientes para incidir en el Bienestar de las personas, por ello la propuesta de integrar sistemas de información centrados en la calidad de vida y salud social de la población, más que en la medición de la producción económica.

La medición del bienestar subjetivo debe sumarse también a los indicadores de Calidad de Vida de la población, sobre todo por sus implicaciones en la Salud, entendida — como lo establece la Organización Mundial de la Salud desde su constitución en 1948-, como un estado pleno de bienestar, con la finalidad de verificar que los indicadores de bienestar a nivel macro, se ven reflejados en el bienestar de la gente y por ende en sus niveles de calidad de vida.

\section{Referencias}

Beltrán Guerra, L.F., Arellanez Hernández J.L., Romero Pedraza, E. (2017). Diagnóstico de la Juventud. Informe de investigación. Universidad Veracruzana

Beltrán Guerra, L.F. (2018). La psicología social en la medición del bienestar subjetivo para gestionar el desarrollo de las sociedades. Psicología Social y Cultura. México: Universidad Veracruzana.

Goldberg, D., Bridges, K., Duncan-Jones, P. y Grayson, D. (1988). Detecting anxiety and depression in general medical settings. BMJ Clinical Research 297(6653), 897-899.

Happy Planet Index (2020). Happy Planet Index México. Recuperado de: http://happyplanetindex.org/countries/mexico

Naciones Unidas (2018), La Agenda 2030 y los Objetivos de Desarrollo Sostenible: una oportunidad para América Latina y el Caribe (LC/G.2681-P/Rev.3), Santiago. 
Recuperado de:

https://repositorio.cepal.org/bitstream/handle/11362/40155/24/S1801141_es.pdf

Nussbaum, Martha C. y Sen, Amartya. (Comp.) (2000). La calidad de vida. México: Fondo de Cultura Económico \& The United Nations University.

Programa de las Naciones Unidas para el Desarrollo (2020). Índice de Desarrollo humano.

Recuperado de: http://hdr.undp.org/en/home

Organización Mundial de la Salud (1948). Constitución de la Organización Mundial de la

Salud. Recuperado de: https://apps.who.int/gb/bd/PDF/bd47/SP/constitucionsp.pdf?ua=1

Rojas, Mariano (2011). Más allá del ingreso: progreso y bienestar subjetivo. En Rojas, Mariano (Coord.). (2011). La medición del progreso y del bienestar. Propuesta desde América Latina. México: Foro Consultivo, Científico y Tecnológico A.C. ISBN: 978-607-95050-6-6 Recuperado de:

http://www.foroconsultivo.org.mx/libros_editados/midiendo_el_progreso_2011_esp.p df

Rojas, Mariano (2012) (Coord.) Medición, investigación e incorporación a la política pública del bienestar subjetivo: América Latina. Reporte de la Comisión para el Estudio y la Promoción del Bienestar en América Latina. Foro Consultivo, Científico y Tecnológico A.C. ISBN: 978-607-9217-10-5 Recuperado de:

http://www.foroconsultivo.org.mx/libros_editados/midiendo_el_progreso_reporte_co mision_esp.pdf

Secretaría de Salud (1983a). Reglamento de la Ley General de Salud en Materia de

Investigación para la Salud. Diario Oficial de la Federación. Consultado el 12 de julio de 2019. Recuperado de:

http://www.salud.gob.mx/unidades/cdi/nom/compi/rlgsmis.html

Secretaría de Salud (2013b). NORMA Oficial Mexicana NOM-012-SSA3-2012, Que establece los criterios para la ejecución de proyectos de investigación para la salud en seres humanos. Diario Oficial de la Federación. Recuperado de: https://dof.gob.mx/nota_detalle.php?codigo=5284148\&fecha=04/01/2013

Sen, A., Stiglitz, J., Fitoussi, J. (2009). Informe de la Comisión para la Medición del Desempeño Económico y Progreso Social. Recuperado de: www.stiglitz-sen-fitoussi.fr

Sociedad Mexicana de Psicología (2009). Código Ético del Psicólogo. Cuarta edición. México: Trillas. 